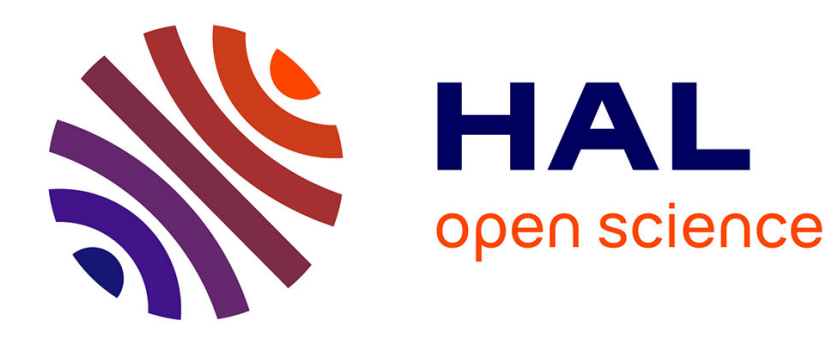

\title{
Syndicalistes croyants et panafricains
}

Françoise Blum

\section{To cite this version:}

Françoise Blum. Syndicalistes croyants et panafricains: Réseaux des années 60. Vingtième siècle. Revue d'histoire, 2013, 3, pp.99-112. halshs-00856488

\section{HAL Id: halshs-00856488 \\ https://shs.hal.science/halshs-00856488}

Submitted on 1 Sep 2013

HAL is a multi-disciplinary open access archive for the deposit and dissemination of scientific research documents, whether they are published or not. The documents may come from teaching and research institutions in France or abroad, or from public or private research centers.
L'archive ouverte pluridisciplinaire $\mathbf{H A L}$, est destinée au dépôt et à la diffusion de documents scientifiques de niveau recherche, publiés ou non, émanant des établissements d'enseignement et de recherche français ou étrangers, des laboratoires publics ou privés. 
Syndicalistes croyants et panafricains : réseaux des années $60^{1}$

Par Françoise Blum (Centre d'histoire sociale du XXe siècle)

«Pour des raisons de fraternité et de solidarité humaine, mais aussi pour des raisons d'équilibre des forces, -de maintien de l'emploi- les hommes de 1968 doivent résoudre le problème du développement... »

" Après l'indépendance, les ouvriers embrigadés dans les syndicats ont l'impression de tourner à vide. L'objectif limité qu'ils s'étaient fixé se révèle, au moment même où il est atteint, bien précaire eu égard à l'immensité de la tâche de construction nationale »(Frantz Fanon, Les Damnés de la terre)

Le processus de balkanisation politique de l'Afrique, largement initié par la loi-cadre de 1956, est maintenant connu dans ses grandes lignes. La balkanisation syndicale, par contre, n'a jusqu'ici suscité que peu de travaux ${ }^{2}$. Comme en ont peu suscité les tentatives de recompositions panafricaines. Nous essaierons dans cet article d'apporter quelques lueurs sur ces phénomènes, à travers l'expérience du syndicalisme croyant. Plus qu'aux organisations, telles la Confédération Africaine des Travailleurs croyants (CATC) puis l'Union Panafricaine des Travailleurs Croyants (UPTC) et la Confédération Syndicale Africaine (CSA), nous nous intéresserons ici aux réseaux des travailleurs croyants, à ces militants qui, d'un bout à l'autre de l'ex-empire français, font vivre un panafricanisme de fait, tout en rêvant d'une Afrique unie sinon unifiée. Ils sont confrontés aux aléas politiques qui secouent le continent et à la dérive générale que constituent l'avènement des partis uniques et son corollaire, l'intégration syndicale au parti, au nom de la nécessité de la construction nationale. Défenseurs de la liberté et de l'autonomie syndicale, fidèles en cela sans peut-être le savoir à la tradition de la Charte d'Amiens ou à celle de la doctrine catholique des corps intermédiaires, ils essaient aussi de penser un syndicalisme africain, au cœur d'une guerre froide dont ils sont l'un des enjeux.

\footnotetext{
${ }^{1}$ Cet article utilise largement le Fonds Gabriel Espéret, conservé aux archives confédérales de la CFDT. Nous remercions Annie Kunhmunch, Elise Mavraganis et Nicolas Perrais de nous en avoir facilité l'accès. Pour ne pas avoir à répéter nous utiliserons la notation GE pour désigner : Archives Confédérales de la CFDT, Fonds Gabriel Espéret.
}

Je remercie également Souleymane Bachir Diagne et Danièle Voldman pour leur relecture attentive.

${ }^{2}$ On peut consulter : Andras November, L'évolution syndicale en Afrique occidentale ; pref. De Gabriel d'Arboussier, Mouton,1965 et Gérard Fonteneau, Histoire du syndicalisme en Afrique, Karthala, 2004 
L'existence légale du syndicalisme est extrêmement tardive dans les territoires d'outre-mer de l'empire français. En Afrique Occidentale Française (AOF), Afrique Equatoriale Française (AEF) et Madagascar, c'est le Front populaire avec les décrets du 11 et 22 mars 1937, qui autorise les travailleurs capables de lire et écrire le français à se syndiquer ${ }^{3}$. La restriction est de taille même si la pratique est plus souple que la loi et même si les luttes sociales et les associations de travailleurs n'ont pas attendu d'autorisation pour exister. Après la deuxième guerre mondiale, les choses s'accélèrent. D'une part, une vague de grèves sans précédent où se mêlent étroitement le politique avec l'aspiration à l'indépendance - et le social - en amont et en aval de l'application du Code du travail de 1952 - secoue le continent. La plus célèbre est la grande grève victorieuse des cheminots en 1947-1948, romancée par Sembène Ousmane dans les Bouts de bois de Dieu ${ }^{4}$. D'autre part, le syndicalisme africain ne peut éviter de reproduire les divisions métropolitaines : Confédération Générale du Travail (CGT), Confédération Française des Travailleurs Chrétiens (CFTC) et à partir de 1948 Confédération Générale du Travail -Force Ouvrière (CGT-FO). Il existe néanmoins très tôt un syndicalisme autonome, avec par exemple la Fédération autonome des syndicats cheminots d'AOF, créée en 1947. Sous l'influence entre autre des partis, cette tendance à l'autonomie va s'accentuer. 1955 est l'année de naissance de la Confédération Générale des Travailleurs Africains (CGTA), qui rompt avec la CGT en 1956. En 1956 également, la Confédération Africaine des Travailleurs Croyants (CATC) naît à Ouagadougou et en 1957, c'est l'Union Générale des Travailleurs d'Afrique Noire (UGTAN) dont le congrès constitutif a lieu le 20 juin 1958 à Dakar ${ }^{5}$. L'UGTAN appelle, à côté de la Fédération des étudiants d'Afrique noire (FEANF) ou du Parti Africain de l'indépendance (PAI), à voter « non » au referendum de $1958^{6}$. Après le referendum - et la victoire du oui -, l'association éclate en trois blocs : I'UGTAN orthodoxe, qui se fait la voix d'un syndicalisme politisé soumis au parti unique ; les UGTAN autonomes sur les différents territoires ; I'UGTAN unitaire qui tente de se fédérer à l'échelle de la Communauté. La tendance CGT-FO n'est pas représentée dans I'UGTAN, à qui les syndicalistes chrétiens font également bientôt défaut en fondant de leur côté l'Union panafricaine des travailleurs croyants (UPTC) qui se veut couvrir l'AOF et l'AEF, sans exclure pour autant les Afrique lusophone et anglophone. Elle souhaite étendre son influence « de la Mauritanie à

\footnotetext{
${ }^{3}$ Voir à ce sujet Nicole Bernard-Duquenet, Le Sénégal et le Front populaire, L'Harmattan, 1985

${ }^{4}$ Sur cette grève, voir notamment Frédérick Cooper, « Our strike : equality, anticolonial politics and the 19471948 railway strike in French West Africa, Journal of African History, 37(1996), p.81-118......

${ }^{5}$ Voir Andras November, L'évolution syndicale en Afrique occidentale, op. cit. Voir aussi : GE-10P

${ }^{6}$ Le vote portait sur la Constitution de 1958, qui remplaçait l'Union française par la Communauté. Seule la Guinée vota non et devint de ce fait indépendante.
} 
Madagascar ». Elle prend de facto la suite à la fois de la CATC et du COSUF, le bureau africain de la Confédération internationale des syndicats chrétiens (CISC ${ }^{7}$.

Tributaire dès sa légalisation des divisions métropolitaines, le syndicalisme africain ne va pas non plus échappé aux clivages de la guerre froide. II va être bientôt divisé en deux blocs. Le premier est constitué par les adhérents de l'Union syndicale panafricaine (USPA), née des suites de la conférence tenue en janvier 1961 à Casablanca ${ }^{8}$, où étaient représentés entre autres des organisations venant du Ghana, de Guinée, du Mali, d'Egypte, du Maroc et le GPRA ${ }^{9}$. La fédération récuse, dans l'esprit de Bandoeng, toute affiliation internationale, mais est proche de la communiste Fédération syndicale mondiale (FSM). La Guinée de Sékou Touré, l'Algérie socialiste et le Ghana de Nkwameh N'krumah en sont les leaders symboliques. Le deuxième groupe est celui des « modérés » qui, mécontents de la tournure prise à Casablanca ${ }^{10}$ organise, du 9 au 14 janvier $1962^{11}$, une contre-conférence à Dakar . Y est créée, sous la présidence du congolais Gilbert Pongault, secrétaire général de l'UPTC, la Confédération Syndicale Africaine (CSA), présidée jusqu'à sa mort en juin 1966 par le tunisien Ahmed Tlili. Le secrétariat en est assuré par le cheminot guinéen David Soumah, exilé au Sénégal, après les persécutions dont il a été victime - sa maison a été incendiée en 1961 par les partisans de Sékou Touré $^{12}$-. Contrairement aux syndicalistes de Casablanca, les délégués de Dakar ne récusent aucunement l'affiliation internationale. L'UPTC notamment est la branche africaine de la Confédération Internationale des Syndicats Chrétiens (CISC). Elle est née lors d'une conférence accueillant, du 11 au 14 janvier 1959, toutes les centrales d'Afrique adhérentes de la CISC. La Confédération Internationale des Syndicats Libres (CISL) a aussi une branche africaine : l'Organisation Régionale Africaine (ORAF). L'originalité de l'Afrique dans le domaine syndical n'est certes pas cette division en courants socialiste, chrétien et « libre " mais réside plutôt dans les tentatives de regroupements à l'échelle du continent, au-delà des tendances. En 1964 , la CSA et

\footnotetext{
${ }^{7}$ Voir Andras November, op.cit.

${ }^{8}$ Sur la conférence de Casablanca voir : A. November, op.cit.On trouve également beaucoup d'informations dans GE - 10P72

${ }^{9}$ Pays représentés à Casablanca

${ }^{10}$ Id.

${ }^{11}$ Cf November, op.cit. et GE-10P72

${ }^{12}$ GE - 10P5 - Lettre de GE à Madame Soumah (1984) : « Je me souviens du courage que vous avez eu pour aller trouver Sékou Touré après que ses troupes eurent brûlé votre maison à Conakry. J'ai su aussi que la vie n'avait pas toujours été facile pour vous à Dakar » .
} 
I'USPA $^{13}$ réussissent à organiser un congrès commun, témoignant d'un réel élan panafricain, d'autant plus peut-être que les libertés syndicales sont alors battues en brèche par les nationalismes politiques . Le panafricanisme peut être une des formes de la résistance à la mise au pas des syndicats par ces partis uniques, qui sont les fers de lance des constructions nationales.

Ce bref tableau de la situation permet de situer le syndicalisme croyant dans un environnement plus large, où l'Afrique est un des champs de bataille de la guerre froide. Nous allons tenter d'en analyser les aspects doctrinaux, d'en dessiner les réseaux africains, franco-africains avec la CFTC puis, à partir de 1964 la CFDT française ${ }^{14}$, et internationaux, avec la CISC devenue suite au processus de déconfessionnalisation la Confédération Mondiale du Travail (CMT) en $1968^{15}$.

Structures et doctrines de l'UPTC sont inscrites dans la déclaration d'intention et les statuts promulgués lors du $1^{\mathrm{er}}$ congrès, tenu à Cotonou du 2 au 5 mai $1960^{16}$. La déclaration de principe ${ }^{17}$, résolument " anti matérialiste », ce qui signifie anti marxiste, inscrit l'homme " créature de Dieu » au centre d'une idéologie du progrès. Un progrès et un développement qui ne se feront pas sans lui, ni non plus sans une Afrique dont «les paysans, les ouvriers et les intellectuels....doivent puiser à leurs propres sources pour prouver au monde qu'ils sont capables, en tenant compte des valeurs de leurs anciennes structures, de concevoir un système économique et social original adapté aux exigences de l'Afrique Nouvelle, en dehors des théories du capitalisme libéral et de celles du socialisme scientifique». Cette doctrine a un nom : le " communautarisme », qui renvoie directement à la pensée d'un Emmanuel Mounier qui appelait de ses vœux une Révolution personnaliste et communautaire ${ }^{18}$, capable de « rétablir entre les individus le lien qui a été perdu » ${ }^{19}$.

13

${ }^{14}$ Sur la CFTC/CFDT, voir notamment Frank Georgi, Eugène Descamps : chrétien et syndicaliste, L'Atelier, 1997 et Frank Georgi, L'invention de la CFDT 1957-1970 : syndicalisme, catholicisme et politique dans la France de I'expansion, L'Atelier/CNRS Ed., 1995

Sur la $\mathrm{CISC} / \mathrm{CMT}$, voir : Patrick Pasture, Histoire du syndicalisme chrétien international : la difficile recherche d'une troisième voie ; trad. Du néerlandais par Serge Govaert, L'Harmattan, 1999.

${ }^{15}$ GE $10 P 69$

${ }^{16}$ Id.

${ }^{17}$ Statuts - Préambule - GE -10P69

${ }^{18}$ Emmanuel Mounier, Révolution personnaliste et communautaire, Aubier, 1935. 
On retrouve là également cette idée de communauté chrétienne - quand il s'agit de l'Afrique, croyante - qui était au fondement de la doctrine d'Economie et Humanisme. Il est sans doute d'ailleurs conceptuellement plus simple de passer de la notion de famille élargie africaine à celle de communauté des croyants, qu'il ne l'était pour les fondateurs d'Economie et humanisme de passer de la cellule familiale étroite à la communauté chrétienne en actes. La nostalgie des sociétés traditionnelles démantelées par la modernité, qui imprègne les réseaux communautaires peut s'appliquer d'autant mieux à l'Afrique que l'on peut y désigner l'intrusion coloniale comme le vecteur de cette modernité délétère. Le " communautarisme " à I'UPTC se veut aussi la « voie africaine de l'édification nationale », acceptant « un socialisme africain » qui puisse " apporter une contribution valable à condition qu'il respecte toutes les valeurs telles que les apportent les coutumes, les modes de vie et les structures africaines en vue de développer le génie africain au service du progrès et de la prospérité de tous. $"{ }^{20}$ Mais les problèmes de doctrine ont finalement peu d'importance par rapport à une pratique que l'on peut résumer en deux mots : réseaux et formation.

\section{Un réseau panafricain}

L'UPTC a tenu des congrès (2-5 mai 60 à Cotonou, 8-12 janvier 1964 à Brazzaville, 22 novembre 3 décembre 1965 à Léopoldville, 11-16 septembre 67 à Ouagadougou...), où sont votées des résolutions pour soutenir le peuple algérien, contre le pouvoir sud-africain, sur la discrimination raciale et religieuse au Soudan, sur le Sud Ouest africain, sur la situation au Cameroun, sur les " troubles raciaux aux Etats-Unis », pour la libération des colonies portugaises, pour la paix au Vietnam, sur la liberté syndicale, cette dernière témoignant de l'incertitude d'un temps africain tiraillé entre les impératifs de la construction nationale et les droits des travailleurs. Comme l'ONU pour d'autres causes plus politiques, l'OIT est ici pensée à la fois comme source de légitimité et comme garante des libertés syndicales, les conventions internationales du travail 87 et $98^{21}$ signées respectivement par 22 et 21 pays africains faisant ici référence. Les plaintes déposées devant le Comité du BIT pour la liberté syndicale seront d'ailleurs nombreuses dans les années soixante, sans qu'aucun résultat notable n'en ait, semble-t-il, jamais découlé. Le colloque de 1967 sur le syndicalisme et le développement tenu à Léopoldville est franco-anglais. Mais comme tout congrès

\footnotetext{
${ }^{19}$ Denis Pelletier, « Utopie communautaire et sociabilités d'intellectuels en milieu catholique dans les années quarante ", Cahiers de l'IHTP, n²0, 1992, pp. 174 ; Voir aussi : Denis Pelletier, Economie et Humanisme : de I'utopie communautaire au combat pour le Tiers-Monde, 1941-1966, Cerf, 1996.

${ }^{20}$ GE-P69

${ }^{21}$ Il s'agit de la convention sur la liberté syndicale et la protection du droit syndical (1948) et de celle sur le droit d'organisation et de négociation collective.
} 
et colloque, il s'agit d'abord de formaliser par la rencontre physique des réseaux qui couvrent I'Afrique francophone et aspirent à s'étendre à l'Afrique anglophone, dont des observateurs, venant du Nyassaland, de Rodhésie du Nord et du Sud, du Kenya, du Liberia, de Sierra Leone, d’Ouganda, d'Ethiopie, du Basutoland, du Bechanaland et de Gambie ${ }^{22}$, sont invités dès le congrès de Brazzavile début 64. Les militants ayant participé à la fondation ou à l'organisation de l'union viennent de pays à majorité chrétienne, catholique comme le Congo, le Cameroun ${ }^{23}$, le $\operatorname{Togo}^{24}$, la RCA ${ }^{25}$ ou protestante comme Madagascar ${ }^{26}$, ainsi que musulmane comme le Sénégal ${ }^{27}$, le Mali ${ }^{28}$, la Mauritanie $^{29}$, le $\operatorname{Tchad}^{30}$ ou la Guinée ${ }^{31}$, ou mixte comme la Haute-Volta ${ }^{32}$, la Côte d'Ivoire ${ }^{33}$ et le Dahomey $^{34}$ : L'UPTC, dont les référents français - jusqu'en 1964 - ou internationaux - jusqu'en 1968 - sont " chrétiens » est " croyante » par souci d'adaptation aux diverses religions pratiquées en Afrique. Néanmoins, comme l'indiquent les prénoms des militants, elle reste à très large dominante chrétienne. Elle fédère à ses débuts 30 centrales de 9 pays anglophones, de 2 pays lusophones et de

\footnotetext{
${ }^{22}$ Liste des observateurs d'expression anglaise invités au congrès et au colloque de I'UPTC - GE-10P69.

${ }^{23}$ Gabriel Lobe, Joseph M'Bong, Etoah Mboudou, Begueni a Bassa, Pierre Tedondje, Ernest Mongory, Eugène Din Din. GE-10P69-Militants ayant participé à la création et à l'organisation de I'UPTC

${ }^{24}$ Bernard Akakpo, Robert de Souza -Id.

${ }^{25}$ Jean-Richard Sandos, Fidèle Ogbami, Henri Dondra -ld.

${ }^{26}$ Eloï Rakoto (CCSM), Jean de britto, L de G Ranaivo, Ralibera (CCSM), Rakotobe , syndicats chrétiens Fiana, Jules Razafinbahiny, secrétaire d'état affaires étrangères. Id.

${ }^{27}$ Ababacar Thiam, Rémy Bocande . Id.

${ }^{28}$ Emmanuel Coulibaly. Id.

${ }^{29}$ Brahier Diop, Teun Djibrill, Joseph Gaye. Id.

${ }^{30}$ Victor Macot, Antoine Laurence, André Leferre, Félix Adande. Id.

${ }^{31}$ Raymond Faber - David Soumah. Id.

${ }^{32}$ Son Goti Omar, Hema Moulaye Scomb, Paul Ibouldo, Some Ananie, Laurent Bandaogo, Frédéric Guirma. Id.

${ }^{33}$ Soumaoro Vassikiri, Claudine Coffie, Joseph Coffie, Almany Onattara. Id.

${ }^{34}$ Sadeler, Serpos Tidjani, Gontran Rodrigues, Raphael Capo Chichi, Anago Amoussa, Corneille Edoh Coffie, Célestin Oliveira. Id.
} 
19 pays francophones ${ }^{35}$. L'union s'auto-déclare panafricaine. Une résolution votée en 1967 donne une définition de ce que la confédération entend par panafricanisme :

Le congrès : demande aux gouvernements africains de déployer tous les efforts afin de faire cesser toutes les attitudes qui ne répondent pas à l'esprit de l'unité ; exige des autorités que prennent fin la multiplication des monnaies aux effigies de chefs d'état - que la convertibilité des monnaies africaines soit assurée partout en Afrique - que la sécurité soit donnée aux africains en déplacement - que notre tradition d'hospitalité fasse progressivement disparaître toutes les barrières artificielles dressées entre frères ; attire l'attention des autorités et des masses africaines sur les déviations du nationalisme aboutissant au chauvisme et aux conflits entre Etats frères ; Affirme sa foi en l'OUA et demande à tous les Etats de renforcer cet organisme de salut et de le rendre populaire dans l'esprit et le cœur des masses africaines $»^{36}(996)$

Le secrétaire général en est dès l'origine le charismatique Gilbert Pongault. Ce fils d'un chef de village du Nord du Congo, à huit jours de pirogue de Brazzaville, était employé à la radio- télévision quand il fut, en 1949, « converti » au syndicalisme par Gérard Espéret ${ }^{37}$, responsable du secrétariat confédéral à l'Outre-mer de la CFTC et partisan de la 1ere heure de l'autonomie du syndicalisme dans les colonies. Gilbert Pongault créa notamment I'Union des syndicats du Congo d'abord CFTC/CFDT puis indépendant, et l'Union de l'Afrique équatoriale. "Très organisé " et " très fiable " ${ }^{38}$, il acquit rapidement une influence sur tout le continent, et au-delà. A côté de Gilbert Pongault, on trouve quelques autres militants qui seront, bien souvent malgré l'adversité, les piliers congolais de I'UPTC: C'est le cas de Pascal Morembe-Ockyemba, ancien séminariste devenu instituteur, avant de présider I'Union nationale CATC du Congo, et permanent syndical. Après être devenu ministre lors de la révolution de 1963, puis avoir connu quelques vicissitudes pendant la phase radicale de cette même révolution, il aura une carrière exemplaire. Conseiller politique du socialiste et chef de l'Etat congolais Marien N'Gouabi, il sera après l'assassinat de celui-ci en 1977 soutenu par le nouveau chef du gouvernement, Pascal Lissouba, qui lui propose de prendre la direction d'un complexe textile, construit par les Chinois dans le cadre de « l'aide aux pays frères ». Il avait suivi en 1967-1968 un

\footnotetext{
${ }^{35}$ En 1967 le bureau de l'UPTC est ainsi composé : Gilbert Pongault (Secrétaire général), Joseph Ouedraogo (Secrétaire général adjoint), Philip Olanrewaju (secrétaire général-adjoint), Blaise Robel (Trésorier), Brahim Bakas (Trésorier adjoint), Jean Diallo, John Lephole, Mullack Dada, MKM Ceesay, Michel Tina, Ahoue Dossou, membres, GKM Mmusi, Mme Médor, M.Ngouandjia, Walker Anguillet, Rauf Adisa Ramos, Eugène Akpemado, membres suppléants - GE -10P69

${ }^{36}$ Illé congrès de l'UPTC. Résolution sur l'unité africaine. GE -10P69

${ }^{37}$ Voir notice de Frank Georgi dans DBMOF : http://maitron-en-ligne.univ-paris1.fr

${ }^{38}$ GE. 10P5. Relations entre Gérard Espéret et des syndicalistes africains affiliés à la CFTC et la CFDT. Notes de GE.
} 
stage de formation à Dairen, en Chine dont il rapporte ses impressions. Outre qu'il profite de l'occasion pour étudier la Bible ${ }^{39}$, et qu'il y est surpris par la conscience professionnelle et la moralité, ainsi que par les aspects d'un communisme chinois où " Mao est Dieu », Il y assiste aux débuts de la révolution culturelle. Il verra le directeur de l'usine où il effectue son stage, un panneau sur le ventre, subir la critique de ses ouvriers et être envoyé en rééducation comme balayeur. II rencontre Mao dont il serre vigoureusement la main « pour s'assurer de sa solidité ». Il sera nommé en 1973 ambassadeur en Chine alors même qu'il est élu député en juin et assure les fonctions de juge à la cour révolutionnaire de justice. En septembre de la même année il devient premier vice-président de l'assemblée nationale populaire, et président du comité exécutif du conseil municipal de Brazzaville. L'importance des militants congolais à l'UPTC s'explique par plusieurs facteurs. Le Congo est très largement chrétien et par conséquent le syndicalisme chrétien, qui y regroupe en 1960 75\% des syndiqués, y trouve une terre prédestinée. Il y aurait eu déjà en 195527 syndicats CFTC ${ }^{40}$. Brazzaville a été de surcroît capitale de l'AEF, ce qui n'est d'ailleurs pas sans lien avec l'importance des richesses du pays, ni avec sa proximité du « grand » Congo belge, objet de toutes les convoitises, dont la largeur d'un fleuve seule la sépare, et dont le représentant au sein de l'UPTC est André Bo-Boliko. Le siège de l'UPTC est d'abord fixé à Brazzaville. Mais la révolution congolaise va changer la situation. En aout 1963, l'abbé Youlou, fantasque ecclésiastique à la tête d'un gouvernement corrompu, est renversé , au cours de ce que l'on appellera « les Trois glorieuses " ${ }^{41}$ (11-14 aout 1963) suite à une grève générale initiée par des syndicalistes qui appartiennent tant à la CGAT de tendance communiste, qu'à la CATC croyante. Bien qu'opposés au cumul des fonctions syndicales et politiques, les travailleurs croyants pensent un moment à une possible présidence de Gilbert Pongault, pour finalement se contenter d'un ministère pour Pascal Ockyemba :

\footnotetext{
${ }^{39}$ « A profité de l'occasion pour étudier la Bible et pour réexaminer l'action passée. Etait là-bas avec 22 congolais dont 2 filles, tous catholiques, protestants ou témoins de Jéhovah »GE. 10P5. Camarades d'Afrique en correspondance. Ces informations biographiques ont été recueillies par Gérard Espéret.

${ }^{40}$ Union fédérale AEF Cameroun, Fédération générale des fonctionnaires d'AEF, Union territoriale CFTC de Pointe-Noire, Syndicat des fonctionnaires, des employés et ouvriers du commerce, des décisionnaires et journaliers, des services publics, du Bois et bâtiment, du personnel civil de l'administration militaire, des brasseries, de la métallurgie, de la météo, etc... -GE -10P80 (Dossier Gilbert Pongault)

${ }^{41}$ Sur la révolution congolaise, voir notamment : Rémy Boutet, Les Trois glorieuses ou la chute de Fulbert Youlou, Chaka, 1990. Deux travaux universitaires sont actuellement en cours sur la révolution congolaise par Héloïse Kiriakou (Cemaf) et Matt Swangler (Columbia).
} 
«Après avoir étudié tous les aspects de notre révolution, nous sommes convenus d'introduire notre ami Pascal Ockyemba dans le gouvernement définitif pour jouer le rôle d'orientation de conception et de contrôle des objectifs de la révolution. Dans cet ordre d'idée, nous avons pensé qu'il devrait être premier ministre [Pascal Ockyemba sera en fait Ministre de la justice]. C'est le seul syndicaliste qui fera partie de notre nouveau gouvernement. Un homme capable d'incarner les aspirations profondes du peuple qui nous surveille. Voila en quelques mots, les raisons qui nous ont poussé à mettre Ockyemba dans le gouvernement.... ...Notre ami Massemba-Débat sera porté à la Présidence de la République.... ${ }^{42}$

Mais, alors même que la révolution s'était faite contre le parti unique voulu par l'abbé Youlou, on en revient au parti unique avec le Mouvement national de la Révolution (MNR), et à son corollaire l'intégration syndicale, inacceptable pour les syndicalistes chrétiens, qui dénoncent une situation où le syndicat n'est plus qu'une simple « courroie de transmission ${ }^{43}$. Et surtout, le gouvernement de Massemba-Debat est bientôt débordé par une jeunesse ${ }^{44}$ qui a fourni les troupes de choc de la révolution, et qui aspire à un monde nouveau où les femmes et les jeunes auraient leur place, un monde en rupture complète avec les temps coloniaux. Cette jeunesse qui se radicalise au cours de la révolution ressemble par certains aspects aux gardes rouges de la révolution culturelle ou aux khmers rouges. Elle porte, peut-être, sur ses épaules l'humiliation des pères, et en tous cas ne les comprend plus. Le marxisme de la JMNR (Jeunesse du mouvement national révolutionnaire), quand il n'est pas teinté de guevarisme, emprunte ses références à la Chine maoïste, qui fournit le modèle exemplaire de la seule révolution paysanne victorieuse jamais enregistrée dans l'histoire du monde. La CATC est interdite, le local de l'UPTC est mis à sac par la JMNR. Les militants de l'UPTC - le camerounais Henri Dondra, et le centrafricain Marcel Begueni a Bassa, le Gabonais Richard N’Zogue, Gilbert Pongault - sont obligés de s'exiler, fuite facilitée par la proximité du CongoLéopoldville. Gilbert Pongault, brièvement arrêté le 27 aout 1963 sous l'accusation d'avoir reçu un

\footnotetext{
${ }^{42}$ Lettre de Gilbert Pongault à Gérard Espéret, le 18 décembre 1963 - GE-10P80 (Dossier Gilbert Pongault)

${ }^{43}$ Mémoire de Gilbert Pongault, Secrétaire général de l'Union panafricaine des travailleurs croyants africains et malgaches sur l'arrestation des syndicalistes CATC à Brazzaville et sur sa condamnation à mort par contumace par le Tribunal populaire de Brazzaville. GE - 10P89 (Dossier Gilbert Pongault)

${ }^{44}$ "...ce camarade m'a laissé entendre que la situation politique est complètement pourrie, que la JMNR est en train de terroriser la population de Brazzaville. Il y a même des filles de treize ans qui portent des armes. D’après lui, la population est vraiment dégoûtée et il est possible, par une réaction de désespoir que I'ensemble de la population s'expose aux mitraillettes de la JMNR. Il prétend que Massemba-Débat lui-même est dépassé. » Lettre de Gilbert Pongault à Gérard Espéret, Lagos, 13 aout 1965 - GE 10P80 (Dossier Gilbert Pongault).
} 
télégramme crypté de Jacques Foccart, sera condamné à mort par contumace « pour complot contre la sécurité intérieure et extérieure de l'Etat et pour trafic d'armes ${ }^{45}$ Le béninois Gontran Rodrigues, ne pouvant plus rien faire pour I'UPTC à Brazzaville, rentre dans son pays ${ }^{46}$. Fulgence Biyaoula est intercepté alors qu'il tentait de s'enfuir déguisé en femme ${ }^{47}$. Beaucoup vont croupir dans les geôles congolaises, et être jugés pour complots avec l'étranger ou menées contre-révolutionnaires par des tribunaux populaires, qui n'offrent guère de garanties en termes de droit. Le siège de la centrale est transféré alors à Lagos, en décembre 1964 avec l'aide du nigérian Bernard Agbator. Mais des conflits répétés avec le Nigerian Workers Congress vont conduire à déménager de nouveau, à Léopoldville/Kinshasa ${ }^{48}$ cette fois puis à Bathurst, où l'UPTC sera finalement dissoute en 1975. Outre les congolais, sévèrement déstabilisés par les évènements, quelques autres figures syndicales vont continuer à faire vivre I'UPTC, comme ils font vivre les centrales de leurs pays réciproques. Ils sont tous au premier rang de l'histoire de leur pays et du continent. Prenons par exemple le sénégalais Ababacar Sadikhe Thiam, au prénom étrangement musulman à côté de ceux, chrétiens, de ses camarades. Ababacar Thiam est raflé comme la grande majorité des leaders syndicaux, à la bourse du travail de Dakar, lors des événements qui secouent le Sénégal en 1968, rafle à laquelle David Soumah échappe par miracle ${ }^{49}$. Son destin ou son itinéraire familial est intéressant, en amont. Originaire de Saint-Louis du Sénégal, l'une des 4 communes, il est né citoyen français. Ses deux grand -pères ont été médaillés des diverses expositions universelles et des arts décoratifs ${ }^{50}$. L'un, Jean Thiam, a été fait chevalier de la légion d'honneur. On trouve A. Thiam au côté de Lamine Gueye à la SFIO. Il est en même temps président du conseil de la jeunesse du Sénégal. Mais il est permanent syndical au Togo, secrétaire général adjoint de la CFTC-AOF-Togo, secrétaire général de la fédération postale AOF-Togo puis de la Fédération des services publics et de l'enseignement CFTC-Togo etc..... ${ }^{51} \mathrm{Ce}$ parcours n'est pas une exception : fonctionnaires et autres employés étaient nommés

\footnotetext{
${ }^{45}$ Mémoire de Gilbert Pongault, op.cit. GE 10 P80 (Dossier Gilbert Pongault)

${ }^{46} \mathrm{GE}$ - 10P5- Dossier Gontran Rodrigues

${ }^{47}$ Coupure de presse (avec photo de l'intéressé en femme) non datée - GE 10P80 (Dossier Gilbert Pongault)

${ }^{48}$ Léopoldville est rebaptisé Kinshasa en 1966.

49 Voir Françoise Blum, « Mai 68 au Sénégal », Revue d’histoire moderne et contemporaine, à paraître

${ }^{50} \mathrm{Gd}$-père maître bijoutier, médaillé et diplômé de l'expo de 1900 ett de celle de Marseille en 1906,en 1922, médaillé d'argent de l'expo des arts décoratifs, industriels et modernes de 1925, autre grand-père Jean Thiam également médaillé à diverses expositions chevalier de la légion d'honneur.GE- 10P5 - Dossier Ababacar Thiam.

${ }^{51}$ GE-10P5 - Dossier Ababacar Thiam.
} 
d'un bout à l'autre de l'AOF et de l'AEF ${ }^{52}$. Et ce sont ces nominations indifféremment faites à travers l'empire qui ont, tout au moins dans cette génération née aux environs de la première guerre mondiale, contribué à forger un panafricanisme de fait, une sensibilité panafricaine assez peu sensible aux frontières. Si Marcel Begueni a Bassa a aussi en lui cet habitus panafricain, c'est pour des raisons plus brutales : responsable du syndicalisme CFTC en République centrafricaine où il fait « un excellent travail ", il est expulsé du pays suite à la " plainte d'un blanc ». Il fonde ensuite l'école des cadres à Brazzaville, pour en être chassé par la révolution. Il se retrouve responsable des problèmes juridiques pour la centrale chrétienne du Cameroun ${ }^{53}$. Joseph Ouedraogo, secrétaire régional de I'UPTC pour l'Afrique occidentale a travaillé un temps à Dakar. Rentré en Haute Volta, son pays natal, il prend la direction de l'union des syndicats et en fera une forte organisation. II sera maire de Ouagadougou, puis Ministre des finances. Arrêté et déporté il sera libéré par le gouvernement du capitaine Sankara « parce que Joseph était à la tête de la révolte populaire qui a mis dehors Maurice Yameogo ${ }^{54}$.

Nous avons là incontestablement une génération militante, qui a forgé ses armes sous la colonisation, ou pour mieux dire une élite militante. Ces hommes ${ }^{55}$ passent du syndical au politique et réciproquement ${ }^{56}$, quelque soit en la matière les réticences syndicales croyantes, -quand ils ne concilient pas les deux -. Ils passent de la clandestinité, de la prison ou de l'exil aux honneurs et des honneurs à l'oubli, et cumulent diverses fonctions.

Ils sont aussi aux avant-postes de révoltes et de ruptures qui parfois les débordent ou les brisent. Destins panafricains, étonnants et chaotiques, voués de toutes façons, malgré tout, à la défense des

\footnotetext{
${ }^{52}$ Voir notamment à ce sujet : Pascale Barthélémy, Africaines et diplômées à l'époque coloniale (1918-1957), Presses universitaires de Rennes, 2010

${ }^{53} \mathrm{GE}-10$ P5 - Dossier Begueni a Bassa.

${ }^{54}$ Id. Dossier Joseph Ouedraogo -

${ }^{55}$ On ne trouve que très peu de femmes parmi eux : Claudine Coffie et la malgache Angélique Médor (membre suppléante du bureau)

${ }^{56}$ En 1962, par exemple voici quelques syndicalistes devenus hommes d’Etat: Mamadou Dia (CGT), Maurice Yameogo (Dahomey -CATC), Sekou Toure (Guinée-UGTAN), Modibo Keita (Mali-CGT), Bacary Djibo (CGT), Assale (CGT puis FO), Ministres : Avaro (CATC), Okomba (CATC), N'Got (CGAT), Gris Camille (UGTAN), Ravarison (Madagascar-CCSM), Douzima (RCA-CATC) ; Ambassadeurs : Alioune Cissé (Sénagal-UGTAN Autonome), Abdoulaye Diallo (Mali-UGTAN Orthodoxe), Fankan (Côte d'Ivoire -CGT Cheminots), Guirma (Haute-VoltaCATC) , Lazare Coulibaly (Mali-UGTAN), Ayoune (Gabon-CATC), Damasse (Gabon-CATC), Hautes fonctionnaires : Jules Razafimbahiny (CCSM-Secrétaire général de l'Organisation économique africaine et malgache) - GE10P69-Note
} 
droits des travailleurs et au développement du continent noir. La génération suivante qui elle, n’a pas connu, ou si peu, la colonisation ne les comprendra bien souvent plus. Elle verra en eux, parfois, des suppôts des puissances coloniales, à cause des liens gardés avec les anciennes métropoles. Victimes de luttes qui les dépasse ou combattants qui ont essayé de donner à l'Afrique sa liberté, ils ont tenté aussi de servir les intérêts d'une classe ouvrière minoritaire dans des pays à large dominante rurale.

\section{Réseaux internationaux}

\section{CFTC/CFDT et CISC (CMT)}

Parmi les chefs d'accusation retenus contre les syndicalistes de la CNTC et de l'UPTC lors de la révolution congolaise figure en bonne place celui de recevoir de l'argent de l'étranger. De fait, les salaires des permanents comme les dotations diverses en matériel viennent largement d'Europe, les cotisations recueillies sur place étant dérisoires. En 1960, le fonds de solidarité de la CISC consacre 94.800 NF aux salaires des permanents de 15 pays africains ainsi qu'au secrétariat de l'UPTC, et ce sur un total de 150000 NF nécessaires. Le salaire d'un permanent est environ de 20000 fcs CFA mensuel et le secrétariat de l'UPTC coûte à lui seul 1.151.960 CFA au début des années $1960^{57}$ (811). En 1967 la subvention accordée par le fonds de solidarité de l'UPTC est de 2965000 francs belges et 135000 pour la CSA $^{58}$. Deux européens sont en charge du secteur Afrique de l'UPTC. Il s'agit du français Gérard Espéret, de la CFDT/CFTC, responsable des pays francophones et du belge Jean Brück responsable de l'Afrique anglophone et des pays ex-belges (Congo-Léo, Rwanda, Burundi) et grand ordonnateur du fonds de solidarité de la CISC/CMT. Jean Brück avait été l'acteur principal de I'autonomisation de la centrale congolaise du Congo-Léopoldville par rapport à la CSC belge. Ils sont tous deux imprégnés de ce tiers-mondisme catholique, qui fait de la solidarité le principe d'un nouvel ordre économique international, fondé sur un « développement harmonisé », et dont le laboratoire a été Economie et Humanisme et le représentant archétypal le père Lebret ${ }^{59}$. Gérard Espéret est d'ailleurs un de ses amis proches ${ }^{60}$. Jean Brück et Gérard Espéret jouent donc un rôle clef à l'UPTC,

\footnotetext{
${ }^{57}$ Propositions au fonds de solidarité pour l'Afrique - GE- 10 P69

${ }^{58} \mathrm{GE}-10 \mathrm{P} 69$ - Subvention Afrique

${ }^{59}$ Rapport financier, Congrès de Brazzavile, 1964 - GE10P69

${ }^{60}$ GE - 10P5 - Dossier Ababacar Thiam - Lettre du 17 janvier 1994 : « J'ai longtemps travaillé avec le Père Lebret qui a préparé un plan pour le Sénégal du temps de Senghor et même avant l'indépendance. Nous étions de vieux amis qui s'étaient rencontrés en 38 et j'ai beaucoup travaillé avec lui dans l'Association Economie et Humanisme... "
} 
affiliée, rappelons-le, à la CISC/CMT. Ils ouvrent largement aux militants africains les portes d'un syndicalisme mondialisé. Car les militants croyants ne sont pas cantonnés à l'Afrique. Ils reçoivent des invitations démultipliées par le contexte de concurrence acharnée de la guerre froide. Le « carnet de route " d'Ababacar Thiam nous montre à quel point les frontières sont poreuses. II dessine une cartographie des déplacements et espaces syndicaux dans le monde, cartographie dans laquelle I'OUA et l'OIT sont d'incontournables carrefours.

« 1960 : secrétaire général adjoint de la confédération africaine des travailleurs chrétiens, basé en Europe où j'ai pris part à divers congrès, séminaires, conférences de même qu'en Afrique, avec un recyclage à Bierville (centre de formation périodique de la CFTC). Mai 61 : Casablanca : conférence constitutive de I'USPA. Juin 61 : ma troisième participation à la conférence annuelle de l'OIT à Genève en tant qu'observateur de la CISC. Octobre 61 : Nairobi (Kenya) en tant que représentant de I'UPTC sous la présidence de feu Tom Boya, nous avons préparé la première conférence panafricaine de Dakar. Janvier 62 : Conférence panafricaine de Dakar. Avril 62 : a l'invitation du Foreign Office, avec d'autres africains, nous avons visité la Grande-Bretagne, ce beau pays, ses syndicats, ses organisations de jeunesse et ses belles réalisations sociales. Nous avons continué la visite en Allemagne fédérale, dans le même but, toujours très émerveillé. Juin 62 : à l'invitation de l'Allemagne de l'Est, j'ai visité ce pays et ses syndicats. Secrétaire chargé de la presse de la formation syndicale d'où de fréquents voyages en Europe. Novembre 64. Addis-Abbeba Ethiopie, Observateur pour la CISC, décembre 64 : Lagos (Nigeria) . 1ere conférence régionale OIT., observateur CISC. Janvier 64 : Brazzaville, Congo colloque international et congrès UPTC avec sa reconduction au poste de secrétaire adjoint. Octobre 65 : Lagos $1^{\text {er }}$ congrès de la CSA. Décembre 65 : congrès UTC. 1966 : boursier du BIT. Stage à la CGT-FO et CFTC. 1967 : Fribourg (Suisse) séminaire international des services publics de l'enseignement où j'ai été élu du groupe africain. 1968 : deuxième conférence régionale de I'OIT à Dakar, observateur UPTC. 1969 : cinquantenaire de I'OIT à Genève, observateur de la CISC. 1971 : Rome, colloque, pèlerinage et audience solennelle du Pape. Cette énumération rappelle seulement quelques principaux colloques, congrès conférences séminaires...sans compter les autres...qui m'ont amené à Malte, aux Pays Bas, au Luxembourg, dans les pays scandinaves. Juin 1972 : a l'invitation des syndicats américains j'ai visité ce vaste et beau pays pendant 45 jours en prenant part à différents colloques, séminaires etc. 1969 : Banjul. Elu président de la fédération panafricaine de l'enseignement et des services publics (AFROFEDOP). Novembre 69 : Bruxelles. Elu vice-président de la Fédération internationale chrétienne des syndicats du personnel public (INFEDOP) $1975: 1^{\text {er }}$ mai, Moscou. Voyage à travers le pays pendant 15 jours où j'ai visité les syndicats et le parti. 1978 : Washington : Colloque international de haute technicité sur les télécommunications. De 1952 à 1972, j'ai participé à tous les congrès et colloques de la CFTC et de la CISC qui ont changé de sigle en 69 pour s'appeler CFDT et CMT ${ }^{61}$.

\section{Coopération}

Ces réseaux panafricains et internationaux participent d'un courant général alors qui inscrit la coopération comme une des missions de "l'Occident", et en ce qui concerne plus spécifiquement la CISC et I'UPTC comme le rachat -concept chrétien- de la colonisation et comme une forme de garantie pour « l'Occident » du droit de jouir en paix des richesses acquises: «Les organisations

\footnotetext{
${ }^{61} \mathrm{GE}$ - 10P5 - Dossier Ababacar Thiam - CV
} 
syndicales sont persuadées que la tension, les sacrifices et la durée nécessairement demandés à I'Afrique pour atteindre rapidement une économie moderne pourront être diminués et abrégés par une coopération internationale représentant la solidarité entre les hommes, les continents et les races. Elles rappellent que cette coopération est à la fois, pour les peuples les plus techniquement avancés, un devoir de solidarité humaine, un devoir de justice réparatrice des prélèvements effectués sous le régime colonial et la sauvegarde des intérêts des peuples, dont le niveau ne pourra être maintenu que s'il est justifié que par cet effort de solidarité humaine $\nu^{62}$

La coopération peut consister, comme nous l'avons vu, en une aide financière directe. Néanmoins le cœur en est constitué par les activités de formation. La doctrine chrétienne a toujours fait de l'éducation et de la formation un élément essentiel. A I'UPTC/CFDT/CISC, cette activité est formalisée au sein d'un organisme original : I'Institut Syndical de Coopération Technique Internationale (ISCTI) ${ }^{63}$, qui s'inscrit dans un paysage mondial où la formation est une technique de propagande comme une autre. Du côté français la CGT a organisé son Université ouvrière ${ }^{64}$. Les soviétiques ont leur université de l'amitié des peuples, les chinois offrent des stages de plus en plus nombreux, Israël n'est pas en reste avec son Institut afro-asiatique, géré par la Histadrout, la Guinée offre ses propres formations syndicales. L'AFL-CIO et la CISL organisent aussi des sessions de formation. L'ISCTI est un organisme cogéré, résultat d'un accord entre des responsables syndicalistes CFDT-CNTCS (Sénégal)- CATC (Congo)- CCSM (Madagascar)-CVT(Vietnam), africains, asiatiques et français. L'ISCTI prend de facto la suite de la section outre-mer de la CFTC, rendue caduque par le vent des indépendances, mais n'est pas intégré au service international de la centrale. L'Institut est déclaré Le 21 mai 62 à la préfecture comme association française, la législation de l'époque le permettant encore, compte tenu des accords conclus entre les pays ayant appartenu à la Communauté. Son existence et son fonctionnement reposent largement sur les épaules de son délégué général Gérard Espéret, par ailleurs secrétaire confédéral. Aussi, quand Gérard Espéret prend en 1967 sa retraite du secrétariat confédéral, et n'assure donc plus le lien institutionnel entre I'ISCTi et la confédération, celle-ci va souhaiter réintégrer l'Institut dans son giron. Après le départ d’Espéret, fort blessé par ce qu'il considère comme un désaveu, en 1970, ce sera chose faite en 1971 avec la création d'une Section coopération à la CFDT, intégrant l'ISCTI. Celui-ci cesse d'exister, en

\footnotetext{
${ }^{62}$ GE- $10 P 69$

${ }^{63}$ L'essentiel de ce qui suit est tiré de GE - 10P50 et 10P51

${ }^{64}$ Les archives en sont conservées à l'Institut d'histoire sociale-CGT . Voir Inventaire en ligne : http://chs.univparis1.fr/Ressources documentaires/Archives africaines.
} 
tous cas sous la forme voulue par ses fondateurs. Le bureau confédéral a émis des doutes sur la bonne adaptation des sessions à l'Afrique. Les financements de l'ISCTI sont assurés pour une part par la CFDT, pour une autre part par le Fonds de solidarité de la CISC et enfin par I'ISCTI lui-même grâce aux cotisations de ses adhérents mais surtout via des aides demandés au Fonds d'Aide à la Coopération (FAC) - qui accorde de 250000 à 500000 francs selon les années ${ }^{65}$-. Outre Gérard Espéret, les hommes de la CFTC/CFDT présents à I'ISCTI sont Maurice Bouladoux ${ }^{66}$, le trésorier -de la CFDT et de I'ISCTI- Jean Alidières, Raymond Lebescond ${ }^{67}$, président du Comité des études, Yves Arcadias secrétaire. A côté d'eux et dès les origines on trouve le syndicaliste vietnamien Tran Quoc Buu et les hommes de I'UPTC, David Soumah président, Gilbert Pongault, Blaise Robel de Madagascar, Charles Mendy du Sénégal, Begueni a Mbassa, André Bo-Boliko. Y seront bientôt cooptés les sud-américains de la Confederacion Latinoamericana de Sindicalistos Cristianaos (CLASC), Miguel Cardoso et Emilio Maspero ${ }^{68}$. Le métissage est aussi la règle pour les enseignants ${ }^{69}$. Ce sont

\footnotetext{
${ }^{65}$ GE-10P50 - Subvention pour la formation des cadres.

${ }^{66}$ Secrétaire général puis président de la CFTC de 1948 à 1953

${ }^{67}$ Raymond Lebescond fut responsable du département formation à la CTC/CFDT de 1952 à 1970 et directeur de l'institut confédéral d'études et de formation syndicales. Voir notice $D B M O F$.
}

${ }^{68}$ En 68, le Psdt en est David Soumah, les vice-psdts Cooptation de : Emilio Maspero (CLASC), Miguel Cardoso (CLASC), André Bo-Boliko, Psdt de l'UNTC,Blaise Robel, secrétaire confédération chrétienne des syndicats malgaches, Réélection comité de gestion :Psdt : David Soumah, Délégué général : GE,Trésorier : J.

Alidières,Secrétaire : Y Arcadias, Vice-Psdts, M. Bouladoux, Emilio Maspero, Gilbert Pongault, ISCTIMembres de I'Institut :Psdt : David Soumah, Psdt de la CSA, Secrétaire grl de l'ONTCS, Délégué grl : Gérard Espéret Vice-psdts Maurice Bouladoux, CFDT,Emilio Maspero, Clasc, Tran Quoc Buu, BATU,GP, UPTC,Trésorier : Jean Alidières, CFDT Secrétaire : Yves Arcadias, CFDT MembresCharles Mendy, trésorier de I'ONTCS Raymond LebscondCharles Monrapha,Martinique,Begueni a Mbassa, CamerounPierre Jeanne , CFDTMiguel Cardoso, CLASC,André BoBoliko, Psdt UNTC Kinshsa,Blaise Robel CCSMS Tana. Conseil d'administration de juin 66David Soumah, Charles Rakotobe, Charles Monrapha, Pierre Jeanne, Tran Quoc Buu, Raymond Lebescond, Charles Mendy, Jean Alidères, GPExcuses : Hattori, Begueni a Mbassa, B. Agbator, M.Boudaloux,Pascal OckyembaObservateurs : J.Diallo, BI. Diame,G.Donbal, A.Kanbire, BI Robel.

Secrétariat : Renée Joubert, Jacques Meindre.

${ }^{69}$ Afr : Blaise Diame, Jean Diallo, N'zogue (Gabon),Ch Rakotobe (Mad), Donat Mutumbo (CCongoKinshsa), Eugène Akpemado (Togo), Alphonse Kambire (Haute-Volta),M. Diarra (Bamako),J Coffie (Côte-d'Ivoire),JB Karagwa (Rwanda),J. Lephole (Lesotho), Philippe Bobandi (Antony), J Ouedraogo (Haute-Volta), Michel Tina (Cameroun), Serpos Tidjani (Dahomey), Dosso Ahowe (Dahomey), Kikongi (UNTC), Cebre Celassie (Addis-Abbeba). GE-10P50 -Liste des enseignants. 
côté africains les syndicalistes croyants, et côté français soit des cédétistes, soit des personnalités expertes proches des doctrines tiers-mondistes en matière de coopération tel René Dumont, ou le socialiste André Philip. La mixité elle-même participe d'une doctrine chrétienne qui conçoit la coopération comme collaborative et intégrée.

L'ISCTI a un triple but : Documentation et études, Information, Formations. La section documentation et études répond aux consultations sur les sujets suivants : économiques, en particulier en matière de planification et développement ; juridiques à propos des constitutions ou révisions constitutionnelles et des projets de loi intéressant les travailleurs; social en matière de salaires ou de sécurité sociale. La section Information publie le bulletin Energies syndicales et répond aux demandes qui appellent une réponse rapide. Enfin, le secteur formation, certainement le plus important, organise différents types de stages. Il y a les sessions régulières sur le territoire français, dans le château de Bierville, propriété de la CFDT. Elles durent 5 semaines et regroupent en général une quarantaine de stagiaires, minoritairement asiatiques et sud-américains, majoritairement africains et en ce sens sont un des lieux possibles de consolidation-formation d'une identité panafricaine. Gérard Espéret en conçoit une satisfaction générale assortie parfois de quelques bémols. II note par exemple à propos de la session tenue à Bierville en 1967 :

« II faut à mon sens être très sensibilisé par le fait que les Africains aiment parler et écouter parler. II faut savoir que cela ne veut pas toujours dire qu'un cours est assimilé. L'on ne perçoit pas, comme avec des stagiaires européens, si l'on se fait comprendre ou si l'on intéresse...Dans le programme actuel, trop chargé, des contacts vrais n'arrivent pas à s'établir entre les sessionnaires; cela est encore plus net entre Africains et Européens, entre Africains et Sud-Américains..... Le problème racial m'a semblé posé...Certains africains ont très ouvertement fait du racisme. Réflexions entendues de nombreuses fois par des camarades Africains s'adressant aux membres du Comité des Etudes non européens : « Vous êtes au service des blancs "...L'ISCTI, $c^{\prime}$ est une affaire CFDT, c'est une affaire française ...ll est apparu très nettement que si aucun des stagiaires n'a contesté ce qui leur était dit en matière de sous-développement des pays du Tiers-Monde, il y avait une gêne sensible à ce que cela soit dit par des Européens. Des réflexions nous faisant part que c'était nous, Européens, qui étions responsables, furent faites, et dans ce terme " Européens ", il n'y avait pas de distinction entre les capitalistes et les syndicalistes, mais pays pauvres face aux pays riches.... ${ }^{70}$

Il y a aussi les formations à la demande qui s'effectuent sur le terrain. Elles peuvent être de base, à titre exceptionnel, comme les « Semaines nationales ouvrières du Cameroun, du Tchad ou du CongoBrazzaville ». Mais le but visé par l'ISCTI est plutôt la formation des formateurs, avec des formations $\mathrm{du} 2^{\mathrm{e}}$ degré effectuées au Sénégal, à Brazzaville, à Madagascar, au Gabon. Etc. Une formation spécifiquement destinée aux travailleuses est organisé à Lomé en 1967, et c'est alors une femme qui en est responsable : la cédétiste Jeannette Laot, alors présidente de la commission féminine de la

\footnotetext{
${ }^{70}$ Réflexions sur la session internationale, Bierville, 1967. GE - 10P51
} 
CFDT. Les formations elles-mêmes ont un contenu doctrinal, avec des cours sur les grandes doctrines - Marxisme, maoïsme, et, bien sûr Communautarisme - et expériences du temps - Socialismes et communismes algériens, cubains, chinois, soviétiques...Elles ont aussi des contenus juridiques - rôle et place des syndicats, délégués d'entreprise etc - et économiques - avec des théories du développement qui offrent une large place à la planification. Malgré la volonté affirmée d'accorder toute son importance au syndicalisme paysan une unique session, semble-t-il lui donne toute sa place : celle organisée dans la ville malgache d'Antsirabe en 1964, où à côté d'un tronc commun deux groupes sont constitués l'un pour les salariés d'entreprise, l'autre pour les salariés agricoles. Les problèmes de l'agriculture malgaches sont largement développés pour le deuxième groupe ${ }^{71}$.. S'il était besoin de démontrer toute la place et l'importance de la formation, et donc de l'ISCTI pour le syndicalisme croyant, il suffirait sans doute de dire que I'UPTC ne lui survivra que 4 ans.

\section{Réseaux d'entraide et d'amitié}

Le syndicaliste Mathieu Dieuloumona ${ }^{72}$, arrêté au moment de la révolution va nommer sa fille Espére. C'est là un symbole qui n'étonnera pas ceux qui connaissent l'Afrique mais en dit néanmoins long sur les liens tissés. Car les réseaux croyants panafricains sont aussi des réseaux d'entraide et d'amitié. Nous avons la chance d'avoir pu consulter les longues correspondances, émaillées d'anecdotes familiales, entre Gérard Espéret et les syndicalistes africains. Mille faits relatés témoignent des liens forts existants, au-delà des questions purement financières. Les évènements liées à la révolution congolaise sont un bon observatoire des aides que peut offrir le réseau dans une adversité fort courante dans l'Afrique des années 1960. Gérard Espéret et Jean Brück s'occupent de trouver et de payer des avocats pour les emprisonnés. Des réseaux d'aide aux familles se mettent également en place, pour assurer sinon les départs du moins la survie ${ }^{73}$. C'est grâce à Henri Dondra que Gérard Espéret réussira à envoyer de l'argent à la famille de Gilbert Pongault, et ce dans une obligatoire clandestinité, puis à la faire quitter le Congo. Si la situation à Brazzaville est alors

\footnotetext{
${ }^{71} \mathrm{GE}-10 \mathrm{P} 51$

${ }^{72} \mathrm{GE}$ - 10P5 - Dossier Mathieu Dieuloumona.

${ }^{73}$ Personnes ayant acceptés de transférer des fonds pour les syndicalistes persécutés au Congo en 1964 - GE$10 \mathrm{P} 80$ (Dossier Gilbert Pongault)
} 
particulièrement critique, elle n'est pas unique. Gérard Espéret s'inquiétera du sort de syndicalistes emprisonnés en Centre-Afrique et écrira à Bokassa pour tenter d'obtenir leur libération ${ }^{74}$.

"Les premières organisations de ce qui devait devenir la confédération africaine des travailleurs croyants puis se fondre dans I'UGTC purent démarrer grâce à plusieurs membres des équipes d'un homme que vous estimez beaucoup, le président Barthélémy Boganda. A l'époque, il était victime de beaucoup de calomnies voir d'arrestations illégales. Il devait devenir un des plus grands hommes d'état d'Afrique et grâce au discours qu'il prononça à Brazzaville à l'adresse du grl de Gaulle, celui qui permit l'indépendance rapide des états africains. Je l'aimai beaucoup ....C'est en raison de ce passé que je me permets de vous demander la libération de mon camarade Jean-Richard Sandos et de mon camarade Malidanga.

Au Sénégal Charles Mendy et Ababacar Thiam seront brièvement emprisonnés en 1968, suscitant les inquiétudes de leur correspondant français. A côté de ces aides que l'on pourrait qualifier de politique, on trouve également toute une série de démarches d'ordre plus personnel. C'est par exemple une aide ponctuelle de $50000 \mathrm{fcs}$ envoyé à l'ancien cheminot Anago Amoussa " ancien stagiaire de Bierville qui vient d'être licencié pour raisons syndicales » (15-2-61). Anago Amoussa est alors à Pointe Noire, qu'il quitte à cause de la révolution pour rejoindre son Bénin natal. S'ensuit une longue correspondance sur les misères de Anago Amoussa, malade hésitant entre la médecine occidentale et les guérisseurs et affligé du salaire de misère de préparateur en pharmacie ${ }^{75}$. Certains sont des habitués de la demande d'argent tel Michel Tina : « un excellent militant au départ, formé en partie par la JOVC. A créé un deuxième syndicat tendance CFDT d'où bagarre. A réussi à ramasser des cotisations. Au moment de la Réalisation obligatoire, il est entré dans le syndicat trop gouvernemental. Ensuite il m'a souvent écrit pour demander de l'argent mais c'était pour lui. Il a fait beaucoup de projets mais toujours il a échoué. II ne lui restait plus qu'à nous apitoyer.... »

Gérard Espéret rend aussi d'autres types de services. II envoie des livres : ceux de Joseph Ki-Zerbo ou les Bouts de bois de Dieu. ${ }^{76}$ II dispense une aide administrative à Célestin Anselme Oliveira de Montaguere, fonctionnaire des PTT béninois, et syndiqué depuis 1945 : « Avons échangé depuis sur la situation au Bénin....Avons remué pas mal de personnes pour lui permettre de porter le nom de son très éloigné ancêtre venu de la région bordelaise [Ollivier au lieu de Oliveira]. Avec du temps, il a récupéré ce nom. Nous avons également rencontré sa fille quand elle était à Toulouse.». ${ }^{77}$

\footnotetext{
${ }^{74}$ Id. Dossier Jean Richard Sandos - Lettre du 5 avril 1974 à Son Excellence Monsieur Le Général Bokassa

${ }^{75} \mathrm{GE}$ - 10P5 - Camarades d'Afrique en Correspondance - Dossier Anago Amoussa -

${ }^{76}$ GE-10P5 - Dossier Montgory.

${ }^{77}$ Id. Camarades d'Afrique en correspondance.
} 
Gérard Espéret sera également sollicité par Ababacar Thiam, désireux de garder une nationalité française dont on lui refuse le certificat. II écrit, entre autres démarches, à Jean Foyer ${ }^{78}$. Ce désir de rester français est consécutif à un problème de retraite, le Sénégal et la France se renvoyant mutuellement la balle. Ce cas est bien loin d'être unique, et au-delà de la question des pensions des anciens combattants, désormais connue, pour ne pas dire médiatisée, mériterait une étude sérieuse. Combien de fonctionnaires ou d'employés ont-ils été ainsi floués de leurs droits à la retraite pour des raisons administratives peu convaincantes, ou à défaut de pouvoir apporter les preuves nécessaires, ou pour cause de la balkanisation? La mobilité au sein de l'empire n'a pas non plus facilité les choses après les décolonisations comme en témoigne le cas de figure suivant rapporté à Gérard Espéret par un de ses correspondants : « Je connais un cas que je te souligne entre mille autres. Un travailleur ayant servi à l'ex-Dahomey a travaillé ensuite en Côte d'Ivoire et a fini sa carrière au Sénégal. II a, à ce titre, bénéficié d'une pension Iprao-Agrom, donc au secteur privé. Pour la liquidation de sa retraite, il a obtenu tous les certificats de travail auprès de ses employeurs antérieurs. L'Iprao pour ne pas rejeter ces attestations a liquidé les périodes d'emploi effectuées au Sénégal et a prétendu s'informer d'abord auprès des autres pays où l'intéressé a travaillé précédemment aux fins de savoir si l'employé a cotisé avant d'enterrer le compte. Il y a de cela plus de 2 ans sans aucune réponse. C'est dire qu'il n'y en aura jamais.... ${ }^{79}$

Ces formes de solidarité sont consubstantielles aux réseaux syndicaux. Elles sont peut-être ici d'autant plus prégnantes qu'elles se greffent sur un habitus africain où l'entraide est une des formes de la survie. Habitus africain mais aussi habitus chrétien, qui fait du don un devoir, ou habitus « communautaire » où l'amitié aristotélicienne est fondatrice du lien entre les personnes ${ }^{80}$. Elles se doublent ici du gouffre entre les pays riches et les pays pauvres, entre les anciens colonisés et les anciens métropolitains, entre " l' Occident » et le Tiers-Monde. En un mot, il s'agit, quelque soient les désirs et bonnes volontés ou le respect mutuel, d'échanges inégaux.

\section{En guise de conclusion}

Actifs politiquement et syndicalement avant les indépendances, les syndicalistes croyants ont continué leur combat dans une Afrique décolonisée. Mais ils ont bien souvent été perçus par la génération montante, largement conquise aux divers « socialismes scientifiques » comme des agents

\footnotetext{
${ }^{78}$ Id. Lettre de GE à Jean Foyer du ... GE -10P5 - Dossier Ababacar Thiam.

${ }^{79}$ GE- $10 \mathrm{P} 5$ 
de l'ancienne puissance coloniale, avec qui ils avaient gardé des liens forts, et dont ils étaient généralement financièrement dépendants. "Les responsables syndicaux qui ont fait leurs armes dans le cadre des formations syndicales métropolitaines ne savent pas organiser les masses urbaines. Ils ont perdu tout contact avec la paysannerie et se préoccupent en premier lieu de l'embrigadement des métallos, des dockers, des fonctionnaires du gaz et de l'électricité. » écrit Frantz Fanon dans les Damnés de la terre. Ils ont effectivement été au service de cette classe ouvrière ultra-minoritaire qui est, en Afrique, une élite.

De leur côté, ils n'ont pas toujours compris les aspirations parfois radicales de cette jeunesse des " années 68 », dont le marxisme est le langage, la Chine ou Cuba les référents. Deux générations se sont confrontées, parfois affrontées, mais persuadées toutes deux de vouloir le bonheur et la liberté de l'Afrique. 